\title{
Unimodular brackets and related structures
}

by

\author{
Wolfgang Rump (Stuttgart)
}

In this paper, we investigate infinite integral matrices $A=\left(a_{i j}\right)_{i, j \in \mathbb{Z}}$ with all $2 \times 2$ minors equal to 1 . We show first that the fraction

$$
\frac{a_{i-1, j}+a_{i+1, j}}{a_{i j}}
$$

is either $\frac{0}{0}$ or integral for all $i, j \in \mathbb{Z}$. For a fixed $i$, this fraction is constant on each interval $j_{1} \leq j \leq j_{2}$ where it is defined. Hence if $a_{i j} \neq 0$ for all $i, j \in \mathbb{Z}$, the value $a_{i, \infty}$ of the above fraction satisfies

$$
a_{i-1, j}+a_{i+1, j}=a_{i, \infty} a_{i j},
$$

and similarly, there exist $a_{\infty, j} \in \mathbb{Z}$ with

$$
a_{i, j-1}+a_{i, j+1}=a_{\infty, j} a_{i j} .
$$

If, without the restriction $a_{i j} \neq 0$, these equations hold for some (necessarily unique) $a_{i, \infty}, a_{\infty, j} \in \mathbb{Z}$, the binary operation $[i, j]:=a_{i j}$ on $\mathbb{Z}$ will be called a unimodular bracket. Thus if we set $[\infty, \infty]:=\infty$, a unimodular bracket can be regarded as a binary operation on $\mathbb{Z} \cup\{\infty\}$. We will show that a unimodular bracket is uniquely determined by the values $[a, \infty],[\infty, b]$ and some element $M \in \mathrm{SL}_{2}(\mathbb{Z})$ (Proposition 3).

The skew-symmetric case

$$
[a, b]+[b, a]=0
$$

will be studied in some detail. Such unimodular brackets are characterized by the equation

$$
[a, b][c, d]-[a, c][b, d]+[a, d][b, c]=0
$$

and a signature $\varepsilon \in\{-1,1\}$ which satisfies $[a+1, a]=\varepsilon$ for all $a \in \mathbb{Z}$ (Proposition 5). Furthermore, they allow a characterization in terms of their signature $\varepsilon$ and the sequence $a_{n}:=[n, \infty]=[\infty, n], n \in \mathbb{Z}$ (Theorem 1 ).

2000 Mathematics Subject Classification: Primary 11B57; Secondary 16D20.

Key words and phrases: Farey sequence, frieze pattern, bimodule. 
A third characterization will be given in terms of the associated Farey sequence (Proposition 6). This implies, in particular, that the classical Farey series [10] give rise to skew-symmetric unimodular brackets.

Farey series, or more generally, periodic Farey sequences, correspond to those skew-symmetric unimodular brackets [, ] which are periodic with respect to both arguments. This implies that the sequence $a_{n}:=[n, \infty]$ is also periodic, while the converse is not true. A periodicity criterion for [, ] in terms of the sequence $\left(a_{n}\right)$ is given in Theorem 2. The special case $a_{n}>0$ $(\forall n \in \mathbb{Z})$ is related to Coxeter's frieze patterns [6]. Conway and Coxeter have shown [5] that frieze patterns are equivalent to triangulated polygons, one of those ubiquitous combinatorial structures like rooted trees [11], nonassociative monomials, lattice paths $([17,3.5])$, etc., encountered in various mathematical structures such as hypersurface cross ratios [13], sign types of root systems $\mathbb{A}_{n}[14,15,16]$, tilting modules of type $\mathbb{A}_{n}[9]$, simply connected algebras of class $\mathbb{A}_{n}[3]$, and so on.

In $\S 4$, we show that bimodules ${ }_{D} B_{E}$ over skew-fields $D, E$ provide an algebraic setting for skew-symmetric unimodular brackets. If the left dimensions $a_{n}$ of the iterated (right resp. left) duals $B^{* n}$ of $B$ are finite, the sequence $\left(a_{n}\right)_{n \in \mathbb{Z}}$ defines a skew-symmetric unimodular bracket [, ] of signature 1 with $a_{n}=[n, \infty]$. There is a hereditary artinian ring $H(B)$ naturally associated to the bimodule $B$ such that all the values of $[$,$] are significant$ for the representation theory of $H(B)$-mod. The Farey sequence $\left(\omega_{n}\right)_{n \in \mathbb{Z}}$ of $[$,$] consists of the dimension vectors of the preprojective and preinjective$ indecomposable $H(B)$-modules, viewed as objects of the derived category $D^{b}(H(B)$-mod $)$. The other values $[a, b]$ of the bracket belong to the horizontal shifts of $\left(\omega_{n}\right)$, which are the Farey sequences of the tilted hereditary rings $H\left(B^{* n}\right), n \in \mathbb{Z}$.

1. Unimodular operations on $\mathbb{Z}$. Consider a binary operation

$$
\mathbb{Z} \times \mathbb{Z} \stackrel{[,]}{\longrightarrow} \mathbb{Z}
$$

on the ring $\mathbb{Z}$ of integers satisfying

$$
[a, b][a+1, b+1]-[a, b+1][a+1, b]=1
$$

for all $a, b \in \mathbb{Z}$. It is convenient to replace the map (1) by an infinite matrix $A=\left(a_{i j}\right)$ over $\mathbb{Z}$, with rows and columns indexed by $\mathbb{Z}$, such that $a_{i j}=[i, j]$ for all $i, j \in \mathbb{Z}$. Then (2) just says that the $2 \times 2$ minors of $A$ are equal to 1 . Let us call such an operation (1) unimodular. Our first observation concerns the opposite operation $[a, b]^{\mathrm{op}}:=[b, a]$.

Proposition 1. The opposite of a unimodular operation is again unimodular.

Proof. This follows immediately from (2). 
Proposition 2. Every unimodular operation (1) satisfies

$$
[a, b+1]([a-1, b]+[a+1, b])=[a, b]([a-1, b+1]+[a+1, b+1])
$$

for all $a, b \in \mathbb{Z}$. Furthermore, $[a, b]$ always divides $[a-1, b]+[a+1, b]$.

Proof. Subtracting (2) from the similar equation

$$
[a-1, b][a, b+1]-[a-1, b+1][a, b]=1,
$$

we get (3). Moreover, (2) implies that $[a, b+1]$ and $[a, b]$ are relatively prime. Therefore, (3) shows that $[a, b]$ divides $[a-1, b]+[a+1, b]$.

In particular, Proposition 2 yields the implication

$$
[a, b]=0 \Rightarrow[a-1, b]+[a+1, b]=0 .
$$

In other words, Proposition 2 states that at least one of the fractions

$$
\frac{[a-1, b]+[a+1, b]}{[a, b]}=\frac{[a-1, b+1]+[a+1, b+1]}{[a, b+1]}
$$

exists and belongs to $\mathbb{Z}$, and that (4) holds with the exception that one of the fractions might be of the form $\frac{0}{0}$. Thus if $[a, b] \neq 0$ for all $b \in \mathbb{Z}$, there is a unique integer $[a, \infty]$ which satisfies

$$
[a-1, b]+[a+1, b]=[a, \infty][a, b]
$$

for all $b \in \mathbb{Z}$. Similarly, if $b$ is kept fixed and $[a, b] \neq 0$ for all $a \in \mathbb{Z}$, Proposition 1 can be applied to give an integer $[\infty, b]$ with

$$
[a, b-1]+[a, b+1]=[\infty, b][a, b]
$$

for all $a \in \mathbb{Z}$.

In general, we can only infer that (5) holds for at most one $[a, \infty] \in \mathbb{Z}$, and similarly for (6). So the occurrence of zeros in the range of the operation (1) might cause some irregularity, which will be excluded from now on. (For a further discussion of irregularities caused by zeros, see Example 3 in $\S 2$.)

Definition 1. A unimodular operation (1) will be called a unimodular bracket if it admits an extension

$$
\widetilde{\mathbb{Z}} \times \widetilde{\mathbb{Z}} \stackrel{[,]}{\longrightarrow} \widetilde{\mathbb{Z}}
$$

to $\widetilde{\mathbb{Z}}:=\mathbb{Z} \cup\{\infty\}$ with $[a, b]=\infty \Leftrightarrow a=b=\infty$ such that (5) and (6) hold.

By the above remarks, such an extension (7) is necessarily unique. On the other hand, the sequences $\left(u_{n}\right)_{n \in \mathbb{Z}}$ and $\left(v_{n}\right)_{n \in \mathbb{Z}}$ given by

$$
u_{n}=[n, \infty], \quad v_{n}=[\infty, n]
$$

determine (7) up to an initial element in $\mathrm{SL}_{2}(\mathbb{Z})$. 
Proposition 3. Let $\left(u_{n}\right)_{n \in \mathbb{Z}}$ and $\left(v_{n}\right)_{n \in \mathbb{Z}}$ be sequences in $\mathbb{Z}$, and $M=$ $\left(\begin{array}{ll}p & q \\ r & s\end{array}\right) \in \mathrm{SL}_{2}(\mathbb{Z})$. Then there exists a unique unimodular bracket $(7)$ which satisfies (8) and

$$
p=[0,0], \quad q=[0,1], \quad r=[1,0], \quad s=[1,1] .
$$

Proof. Starting with the initial matrix $M \in \mathrm{SL}_{2}(\mathbb{Z})$, equation (6) with $a \in\{0,1\}$ can be regarded as a recursive definition of $[0, b]$ and $[1, b]$ for all $b \in \mathbb{Z}$. As (6) implies

$$
\begin{aligned}
\left|\begin{array}{ll}
{[0, b]} & {[0, b+1]} \\
{[1, b]} & {[1, b+1]}
\end{array}\right| & =\left|\begin{array}{ll}
{[0, b]} & v_{b}[0, b]-[0, b-1] \\
{[1, b]} & v_{b}[1, b]-[1, b-1]
\end{array}\right|=-\left|\begin{array}{ll}
{[0, b]} & {[0, b-1]} \\
{[1, b]} & {[1, b-1]}
\end{array}\right| \\
& =\left|\begin{array}{ll}
{[0, b-1]} & {[0, b]} \\
1, b-1] & {[1, b]}
\end{array}\right|
\end{aligned}
$$

induction shows that (2) holds for $a=0$. In this way, the horizontal shifts of $M$ are defined and belong to $\mathrm{SL}_{2}(\mathbb{Z})$. Similarly, (5) yields all vertical shifts of $M$. In the next step, we use (6) to define the horizontal shifts of these vertical shifts. For adjacent vertical shifts of $M$, the horizontal shifts thereof may overlap, but (6) shows that the resulting operation (1) is well defined. By construction, (2) and (6) are satisfied. To show that (5) also holds, it suffices to verify that the recursive rules (5) and (6) commute in the following sense:

$\begin{array}{llllll}{[0,0]} & & {[0,1]} & & {[0,2]} \\ & M & & N & \\ {[1,0]} & & {[1,1]} & & {[1,2]} \\ & P & & Q & \\ {[2,0]} & & {[2,1]} & & {[2,2]}\end{array}$

Starting with $M$, we get $N=\left(\begin{array}{ll}{[0,1]} & {[0,2]} \\ {[1,1]} & {[1,2]}\end{array}\right) \in \mathrm{SL}_{2}(\mathbb{Z})$ by means of $(6)$, and then $Q$ by means of (5). Similarly, we obtain $P$ from $M$ via (5), and we have to show that $P$ yields $Q$ by (6). By induction, this will complete our proof. Now (5) and (6) give

$$
\begin{aligned}
N & =\left(\begin{array}{cc}
{[0,1]} & v_{1}[0,1]-[0,0] \\
{[1,1]} & v_{1}[1,1]-[1,0]
\end{array}\right), \\
Q & =\left(\begin{array}{cc}
{[1,1]} & v_{1}[1,1]-[1,0] \\
u_{1}[1,1]-[0,1] & u_{1} v_{1}[1,1]-u_{1}[1,0]-v_{1}[0,1]+[0,0]
\end{array}\right) .
\end{aligned}
$$

The symmetric shape of $Q$ shows that our claim is true. 
Example 1. The Fibonacci sequence $\left(\varphi_{n}\right)_{n \in \mathbb{N}}$, given by $\varphi_{n-1}+\varphi_{n}=$ $\varphi_{n+1}$ and $\varphi_{1}=\varphi_{2}=1$, extends naturally to a sequence $\left(\varphi_{n}\right)_{n \in \mathbb{Z}}$. By repeated application of the recursive definition, combined with a basic rule for determinants, we get the following equations:

$$
\begin{aligned}
\left|\begin{array}{cc}
\varphi_{n+2} & \varphi_{n} \\
\varphi_{n+4} & \varphi_{n+2}
\end{array}\right| & =\left|\begin{array}{cc}
\varphi_{n+1} & \varphi_{n} \\
\varphi_{n+3} & \varphi_{n+2}
\end{array}\right|=\left|\begin{array}{cc}
\varphi_{n-1} & \varphi_{n} \\
\varphi_{n+1} & \varphi_{n+2}
\end{array}\right|=\left|\begin{array}{cc}
\varphi_{n-1} & \varphi_{n-2} \\
\varphi_{n+1} & \varphi_{n}
\end{array}\right| \\
& =\left|\begin{array}{cc}
\varphi_{n} & \varphi_{n-2} \\
\varphi_{n+2} & \varphi_{n}
\end{array}\right| .
\end{aligned}
$$

For $n \in\{0,1\}$, these determinants are equal to \pm 1 . Hence, by induction, we get

$$
\left|\begin{array}{cc}
\varphi_{n} & \varphi_{n-2} \\
\varphi_{n+2} & \varphi_{n}
\end{array}\right|=(-1)^{n}
$$

for all $n \in \mathbb{Z}$. Moreover, the definition of $\varphi_{n}$ implies that

$$
\varphi_{n-2}+\varphi_{n+2}=3 \varphi_{n}
$$

for all $n \in \mathbb{Z}$. Therefore,

$$
[a, b]:=\varphi_{2(a+b)-1}
$$

defines a unimodular bracket with $[a, \infty]=[\infty, a]=3$ for all $a \in \mathbb{Z}$. Note that the bracket is commutative, and $[a, b]>0$ for all $a, b \in \mathbb{Z}$.

2. The skew-symmetric case. In this section, we consider operations (1) which are skew-symmetric, i.e. which satisfy

$$
[a, b]+[b, a]=0
$$

for all $a, b \in \mathbb{Z}$.

Proposition 4. For every skew-symmetric unimodular bracket (7), there exists a unit $\varepsilon \in \mathbb{Z}^{\times}$such that the equations

$$
\begin{gathered}
{[a+1, a]=\varepsilon} \\
{[a, \infty]=[\infty, a]=\varepsilon \cdot[a+1, a-1]}
\end{gathered}
$$

hold for all $a \in \mathbb{Z}$.

Proof. By (13), we have $[a, a]=0$. Hence, (2) with $b=a-1$ gives

$$
[a, a-1][a+1, a]=1 .
$$

This implies that there is a unit $\varepsilon \in \mathbb{Z}^{\times}$which satisfies (14) for $a \in \mathbb{Z}$. With $b=a-1,(5)$ gives $[a+1, a-1]=[a, \infty] \cdot \varepsilon$. Similarly, with $a=b+1$, (6) gives $[b+1, b-1]=[\infty, b] \cdot \varepsilon$. This proves $(15)$.

In what follows, we call $\varepsilon$ the signature. Note that the opposite bracket $[,]^{\mathrm{op}}$ is of signature $-\varepsilon$. 
THEOREM 1. There is a one-to-one correspondence between sequences of integers $\left(a_{n}\right)_{n \in \mathbb{Z}}$ together with a sign $\varepsilon \in \mathbb{Z}^{\times}$, and skew-symmetric unimodular brackets (7) of signature $\varepsilon$, given by

$$
a_{n}=[n, \infty]=[\infty, n]=\varepsilon \cdot[n+1, n-1] .
$$

Proof. By Proposition 4, every skew-symmetric unimodular bracket with signature $\varepsilon$ defines a sequence $\left(a_{n}\right)$ such that (17) holds. Conversely, Proposition 3 implies that for every sequence $\left(a_{n}\right)_{n \in \mathbb{Z}}$ in $\mathbb{Z}$ together with a sign $\varepsilon \in \mathbb{Z}^{\times}$, there is a unique unimodular bracket $(7)$ with $a_{n}=[n, \infty]=[\infty, n]$ and

$$
\left(\begin{array}{ll}
{[0,0]} & {[0,1]} \\
{[1,0]} & {[1,1]}
\end{array}\right)=\left(\begin{array}{cc}
0 & -\varepsilon \\
\varepsilon & 0
\end{array}\right) .
$$

It remains to show that this bracket is skew-symmetric. Using (5) and (6), we get $[2,0]=a_{1}[1,0]-[0,0]=a_{1} \varepsilon,[2,1]=a_{1}[1,1]-[0,1]=\varepsilon$, and $[1,2]=a_{1}[1,1]-[1,0]=-\varepsilon,[2,2]=a_{1}[2,1]-[2,0]=a_{1} \varepsilon-a_{1} \varepsilon=0$. Hence

$$
\left(\begin{array}{ll}
{[1,1]} & {[1,2]} \\
{[2,1]} & {[2,2]}
\end{array}\right)=\left(\begin{array}{cc}
0 & -\varepsilon \\
\varepsilon & 0
\end{array}\right)
$$

By induction, we obtain

$$
\left(\begin{array}{cc}
{[n, n]} & {[n, n+1]} \\
{[n+1, n]} & {[n+1, n+1]}
\end{array}\right)=\left(\begin{array}{cc}
0 & -\varepsilon \\
\varepsilon & 0
\end{array}\right)
$$

for all $n \in \mathbb{Z}$. Hence (13) holds for $|a-b| \leq 1$. Assume now that for a particular pair $a, b \in \mathbb{Z},(13)$ has been shown. Then (5) and (6) give $([a-1, b]+[b, a-1])+([a+1, b]+[b, a+1])=[a, \infty][a, b]+[\infty, a][b, a]=0$. By induction, this proves (13) for all $a, b \in \mathbb{Z}$.

Our next result shows that apart from the signature condition (14), skewsymmetric unimodular brackets can be characterized by a single equation (cf. $[6, \S 5])$.

Proposition 5. A binary operation (1) satisfying (14) for some fixed $\varepsilon \in \mathbb{Z}^{\times}$is a skew-symmetric unimodular bracket if and only if

$$
[a, b][c, d]-[a, c][b, d]+[a, d][b, c]=0
$$

for all $a, b, c, d \in \mathbb{Z}$.

Proof. Assume first that (18) holds. For $c=d$, this gives $[a, b][c, c]=0$, whence $[c, c]=0$ for all $c \in \mathbb{Z}$. Next, we set $c=a$. This gives $[a, b][a, d]+$ $[a, d][b, a]=0$, which shows that $[$,$] is skew-symmetric. Therefore, the sub-$ stitution $c=a+1, d=b+1$ yields

$$
[a, b][a+1, b+1]-[a, a+1][b, b+1]+[a, b+1][b, a+1]=0 .
$$


Hence

$$
[a, b][a+1, b+1]-[a, b+1][a+1, b]=[a, a+1][b, b+1]=\varepsilon^{2}=1 .
$$

This proves that [, ] is unimodular. Inserting $b=a-1, d=a+1$ into (18), we get

$$
[a, a-1][c, a+1]-[a, c][a-1, a+1]+[a, a+1][a-1, c]=0,
$$

which gives $[a-1, c]+[a+1, c]=\varepsilon[a+1, a-1][a, c]$. Thus (5) holds with $[a, \infty]=\varepsilon[a+1, a-1]$. By the skew-symmetric property, this also implies (6) with $[\infty, b]=\varepsilon[b+1, b-1]$.

Conversely, let [, ] be a skew-symmetric unimodular bracket. Let us keep $b, c, d$ fixed and denote equation (18) by $E(a)$. Assume that $E(a)$ holds for a particular $a \in \mathbb{Z}$. Multiply $E(a)$ by $[a, \infty]$. Then $(5)$ implies that $E(a-1) \Leftrightarrow E(a+1)$. Thus by induction, it suffices to prove $E(b)$ and $E(b+1)$. As $E(b)$ is trivial, we just have to show that (18) holds in the special case $a=b+1$. By a similar reduction, we may also assume that $c=d+1$. So (18) turns into $[b+1, d+1][b, d]-[b+1, d][b, d+1]=1$, that is, the unimodularity condition (2).

Definition 2. A sequence $\left(\omega_{n}\right)_{n \in \mathbb{Z}}$ of vectors $\omega_{n}=\left(\begin{array}{l}x_{n} \\ y_{n}\end{array}\right)$ will be called a Farey sequence if

$$
\left|\begin{array}{ll}
x_{n+1} & x_{n} \\
y_{n+1} & y_{n}
\end{array}\right|=1
$$

for all $n \in \mathbb{Z}$.

Traditionally, for any positive integer $m$, the finite sequence of reduced fractions $\frac{p}{q} \in[0,1]$ with $0<q \leq m$ is called the Farey series $\mathfrak{F}_{m}$ of order $m$. For example, $\mathfrak{F}_{7}$ looks as follows:

$$
\frac{0}{1}, \frac{1}{7}, \frac{1}{6}, \frac{1}{5}, \frac{1}{4}, \frac{2}{7}, \frac{1}{3}, \frac{2}{5}, \frac{3}{7}, \frac{1}{2}, \frac{4}{7}, \frac{3}{5}, \frac{2}{3}, \frac{5}{7}, \frac{3}{4}, \frac{4}{5}, \frac{5}{6}, \frac{6}{7}, \frac{1}{1} .
$$

The remarkable thing is that any pair of successive fractions $\frac{x_{n}}{y_{n}}, \frac{x_{n+1}}{y_{n+1}}$ satisfies (19), while three successive fractions $\frac{a}{b}, \frac{c}{d}, \frac{e}{f}$ are related by $\frac{c}{d}=\frac{a+e}{b+f}$ ([10, Theorems 28 and 29]). Note that every Farey series can be extended to a periodic Farey sequence in the sense of Definition 2. For example, $\mathfrak{F}_{3}=\left\{\frac{0}{1}, \frac{1}{3}, \frac{1}{2}, \frac{2}{3}, \frac{1}{1}\right\}$ gives rise to the Farey sequence

$$
\ldots,\left(\begin{array}{l}
0 \\
1
\end{array}\right),\left(\begin{array}{l}
1 \\
3
\end{array}\right),\left(\begin{array}{l}
1 \\
2
\end{array}\right),\left(\begin{array}{l}
2 \\
3
\end{array}\right),\left(\begin{array}{l}
1 \\
1
\end{array}\right),\left(\begin{array}{l}
1 \\
0
\end{array}\right),\left(\begin{array}{c}
0 \\
-1
\end{array}\right),\left(\begin{array}{l}
-1 \\
-3
\end{array}\right),\left(\begin{array}{l}
-1 \\
-2
\end{array}\right),\left(\begin{array}{l}
-2 \\
-3
\end{array}\right),\left(\begin{array}{c}
-1 \\
-1
\end{array}\right),\left(\begin{array}{c}
-1 \\
0
\end{array}\right), \ldots
$$

of period 12. If we regard these vectors as fractions, the period becomes 6 , and we get $\mathfrak{F}_{3}$ with an additional infinite fraction $\frac{1}{0}$.

By definition, every unimodular operation gives rise to Farey sequences in horizontal and in vertical direction. For a unimodular bracket (7), we 
define its Farey sequence $\left(\omega_{n}\right)$ by

$$
\omega_{n}:=\left(\begin{array}{c}
{[n, 0]} \\
{[n,-1]}
\end{array}\right) .
$$

Proposition 6. A skew-symmetric unimodular bracket (7) is uniquely determined by its signature $\varepsilon$ and its Farey sequence $\left(\omega_{n}\right)$. Explicitly, the bracket is given by the formula

$$
[a, b]=\varepsilon \cdot \operatorname{det}\left(\omega_{a}, \omega_{b}\right) .
$$

Proof. This follows by inserting $c=0, d=-1$ into (18).

REMARK. Note that the Farey sequence (20) always contains the vectors $\omega_{0}=\left(\begin{array}{l}0 \\ \varepsilon\end{array}\right)$ and $\omega_{-1}=\left(\begin{array}{c}-\varepsilon \\ 0\end{array}\right)$. Equation (21) shows that if the Farey sequence $\left(\omega_{n}\right)$ is periodic with period $p$, the bracket [, ] is doubly periodic with period $(p, p)$.

Example 2. A skew-symmetric analogue to Example 1 is given by

$$
[a, b]:=\varphi_{2(a-b)} .
$$

By (10) and (11), this is a (skew-symmetric) unimodular bracket of signature 1 with $[a, \infty]=[\infty, a]=3$ for all $a \in \mathbb{Z}$.

EXAMPLE 3 . The Farey series $\mathfrak{F}_{3}$ gives rise to a doubly periodic skewsymmetric unimodular bracket, consisting of the horizontal and vertical translations of the following matrix:

$$
\begin{array}{rrrrrrrrrrrr}
0 & -1 & -1 & -2 & -1 & -1 & 0 & 1 & 1 & 2 & 1 & 1 \\
1 & 0 & -1 & -3 & -2 & -3 & -1 & 0 & 1 & 3 & 2 & 3 \\
1 & 1 & 0 & -1 & -1 & -2 & -1 & -1 & 0 & 1 & 1 & 2 \\
2 & 3 & 1 & 0 & -1 & -3 & -2 & -3 & -1 & 0 & 1 & 3 \\
1 & 2 & 1 & 1 & 0 & -1 & -1 & -2 & -1 & -1 & 0 & 1 \\
1 & 3 & 2 & 3 & 1 & 0 & -1 & -3 & -2 & -3 & -1 & 0 \\
0 & 1 & 1 & 2 & 1 & 1 & 0 & -1 & -1 & -2 & -1 & -1 \\
-1 & 0 & 1 & 3 & 2 & 3 & 1 & 0 & -1 & -3 & -2 & -3 \\
-1 & -1 & 0 & 1 & 1 & 2 & 1 & 1 & 0 & -1 & -1 & -2 \\
-2 & -3 & -1 & 0 & 1 & 3 & 2 & 3 & 1 & 0 & -1 & -3 \\
-1 & -2 & -1 & -1 & 0 & 1 & 1 & 2 & 1 & 1 & 0 & -1 \\
-1 & -3 & -2 & -3 & -1 & 0 & 1 & 3 & 2 & 3 & 1 & 0
\end{array}
$$

The positive part below the main diagonal of zeros is a frieze pattern in the sense of Coxeter [6]. (To get his pictures, we have to apply a rotation of angle $\pi / 4$ in the positive direction.) The whole range of the bracket consists of an alternating sequence of positive and negative frieze patterns, separated by diagonals of zeros. The conditions (5) and (6) ensure that all these frieze patterns are of the same type. If we drop conditions (5) and (6), we get a multitude of unimodular operations just by patching together arbitrary positive and negative frieze patterns, with the only restriction that adjacent ones differ in sign. 
3. Periodicity. A finite sequence of integers, modulo the equivalence relation generated by

$$
\begin{gathered}
\left(a_{1}, \ldots, a_{n}\right) \sim\left(a_{2}, \ldots, a_{n}, a_{1}\right), \\
\left(a_{1}, \ldots, a_{n}\right) \sim\left(a_{1}, \ldots, a_{n}, a_{1}, \ldots, a_{n}, \ldots, a_{1}, \ldots, a_{n}\right),
\end{gathered}
$$

will be called a cycle. (The right-hand side of the second equivalence has $n \cdot m$ terms, with $m \geq 2$ arbitrary.) Thus a cycle is tantamount to a periodic sequence, up to shift. A cycle $\left(a_{1}, \ldots, a_{n}\right)$ with different terms $a_{i}$ can also be regarded as a cyclic permutation.

Assume that a skew-symmetric unimodular bracket (7) is given. By the remark that follows Proposition 6, such a bracket is doubly periodic with period $(p, p)$ if and only if the associated Farey sequence $\left(\omega_{n}\right)$ is periodic with period $p$. By Theorem 1, the periodicity of $\left(\omega_{n}\right)$ merely depends on the sequence $a_{n}:=[n, \infty]$, and an obvious necessary condition for the periodicity of $\left(\omega_{n}\right)$ is that $\left(a_{n}\right)$ is periodic. However, as Example 2 shows, this condition is not sufficient.

In the present section, we characterize the sequences $\left(a_{n}\right)_{n \in \mathbb{Z}}$ for which $\left(\omega_{n}\right)$ is periodic. For simplicity, we call the bracket itself periodic if its associated Farey sequence is so. If $\left(a_{n}\right)$ has period $p$, the cycle $\left(a_{1}, \ldots, a_{p}\right)$ will be called the characteristic cycle of the bracket. For instance, the bracket of Example 3 has the characteristic cycle $(1,3)$, which could also be written, e.g., as $(3,1,3,1,3,1)$. Note that the characteristic cycle determines the bracket up to signature, and up to translation. Thus it makes sense to call a cycle $\left(a_{1}, \ldots, a_{p}\right)$ of finite type if the skew-symmetric unimodular brackets admitting $\left(a_{1}, \ldots, a_{p}\right)$ as a characteristic cycle are periodic. The set of cycles of finite type will be denoted by $\mathfrak{F}$.

\section{THEOREM 2.}

(a) Every cycle $\left(c_{1}, \ldots, c_{p}\right)$ of finite type contains a term $c_{i}$ with $\left|c_{i}\right| \leq 1$.

(b) $\left(a, 0, b, c_{1}, \ldots, c_{p}\right) \in \mathfrak{F} \Leftrightarrow\left(a+b, c_{1}, \ldots, c_{p}\right) \in \mathfrak{F}$.

(c) $\left(a, 1, b, c_{1}, \ldots, c_{p}\right) \in \mathfrak{F} \Leftrightarrow\left(a-1, b-1, c_{1}, \ldots, c_{p}\right) \in \mathfrak{F}$.

(d) $\left(a,-1, b, c_{1}, \ldots, c_{p}\right) \in \mathfrak{F} \Leftrightarrow\left(a+1, b+1, c_{1}, \ldots, c_{p}\right) \in \mathfrak{F}$.

(e) The cycles $(a, b) \in \mathfrak{F}$ are $(0,0),(1, n),(-1,-n)$ with $n \in\{1,2,3\}$.

Proof. Assume that a skew-symmetric unimodular bracket (7) with associated Farey sequence $\left(\omega_{n}\right)$ and $a_{n}:=[n, \infty]$ admits a characteristic cycle $\left(a_{1}, \ldots, a_{p}\right)$. Consider the matrices

$$
U_{n}:=\left(\begin{array}{cc}
0 & -1 \\
1 & n
\end{array}\right)=\left(\begin{array}{cc}
0 & -1 \\
1 & 0
\end{array}\right)\left(\begin{array}{ll}
1 & n \\
0 & 1
\end{array}\right) \in \mathrm{SL}_{2}(\mathbb{Z})
$$

with $n \in \mathbb{Z}$. By (5) and (20), we have

$$
\left(\omega_{n-1}, \omega_{n}\right) U_{a_{n}}=\left(\omega_{n}, \omega_{n+1}\right) .
$$


Here $\left(\omega_{i}, \omega_{i+1}\right)$ denotes the square matrix with columns $\omega_{i}$ and $\omega_{i+1}$. Thus $\left(a_{1}, \ldots, a_{p}\right)$ is of finite type if and only if

$$
\left(U_{a_{1}} \cdots U_{a_{p}}\right)^{r}=1 \quad \text { for some } r \geq 1 .
$$

To prove (a), let $(x, y)$ denote the second row of the matrix $\left(\omega_{n-1}, \omega_{n}\right)$. Then (24) implies that the second row of $\left(\omega_{n}, \omega_{n+1}\right)$ is $\left(y, a_{n} y-x\right)$. If $\left|a_{n}\right|>1$, we have

$$
\left|a_{n} y-x\right|-|y| \geq\left|a_{n} y\right|-|x|-|y| \geq 2|y|-|x|-|y|=|y|-|x| .
$$

For $n=0$, we have $|x|=0<1=|y|$. Thus if $\left|a_{n}\right|>1$ for all $n$, we infer that the second coordinate of $\omega_{n}$ is unbounded, whence $\left(a_{1}, \ldots, a_{p}\right)$ cannot be of finite type.

The equivalences (b), (c), (d) follow from the identities

$$
U_{a} U_{0} U_{b}=-U_{a+b}, \quad U_{a} U_{1} U_{b}=U_{a-1} U_{b-1}, \quad U_{a} U_{-1} U_{b}=-U_{a+1} U_{b+1},
$$

which are easily verified. To prove (e), suppose that $(a, b) \in \mathfrak{F}$. For $a=0$, we have

$$
U_{0} U_{b} U_{0} U_{b}=-U_{0} U_{2 b}=\left(\begin{array}{cc}
1 & 2 b \\
0 & 1
\end{array}\right),
$$

a translation in $\mathrm{SL}_{2}(\mathbb{Z})$. Hence $(0, b) \in \mathfrak{F} \Leftrightarrow b=0$. For $a=1$, we have

$$
\begin{aligned}
(1, b) \in \mathfrak{F} & \Leftrightarrow(1, b, 1, b) \in \mathfrak{F} \Leftrightarrow(1, b-1, b-1) \in \mathfrak{F} \Leftrightarrow(b-1,1, b-1) \in \mathfrak{F} \\
& \Leftrightarrow(b-2, b-2) \in \mathfrak{F} .
\end{aligned}
$$

By (a), this is possible only if $|b-2| \leq 1$, i.e. $b \in\{1,2,3\}$. Similarly,

$$
(-1, b) \in \mathfrak{F} \Leftrightarrow(b+2, b+2) \in \mathfrak{F} \Rightarrow b \in\{-1,-2,-3\} .
$$

To complete the proof, we only have to note that the order of $U_{1} U_{b}$ or $U_{-1} U_{-b}$ with $b \in\{1,2,3\}$ is 3,4 , or 6 , respectively.

REMARKS. 1. Theorem 2 provides a reduction algorithm which reduces arbitrary cycles to cycles $(a, b)$ with two terms. Moreover, the two-termed cycles of finite type allow a further reduction to $(0,0)$ :

$$
(1,3)=(1,3,1,3,1,3) \stackrel{(\mathrm{c})}{\longrightarrow}(1,3,1,2,2) \stackrel{(\mathrm{c})}{\longrightarrow}(1,2,1,2) \stackrel{(\mathrm{c})}{\longrightarrow}(1,1,1) \stackrel{(\mathrm{c})}{\longrightarrow}(0,0) .
$$

This includes a reduction of $(1,2)=(1,2,1,2)$ and $(1,1)=(1,1,1)$. In a similar fashion, the cycles $(-1, b)$ with $b \in\{-1,-2,-3\}$ reduce to $(0,0)$.

2 . An interesting special case arises when $\left(a_{n}\right)$ consists of positive integers only. The periodic brackets with this property are equivalent to Coxeter's frieze patterns [6] which are in one-to-one correspondence with triangulated polygons [5]. According to Theorem 2, the reduction of such a periodic bracket can be achieved by a single rule (c). This can be visualized by dismantling triangulated polygons. For example, the cycle $(1,3)=$ 
$(1,3,1,3,1,3)$ corresponds to the triangulated hexagon

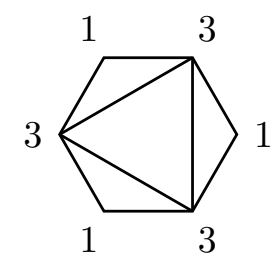

The number at each vertex counts the number of triangles touching that vertex. If a vertex 1 is removed according to rule (c), the triangle at this vertex has to be removed, which implies that the numbers at the two adjacent vertices are diminished by 1 . So the above reduction of the cycle $(1,3)$ looks as follows:

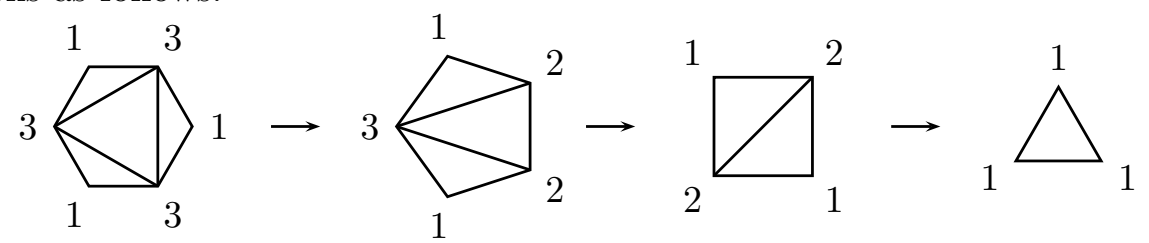

4. Bimodules over skew-fields. In what follows, we give a representation-theoretic interpretation of skew-symmetric unimodular brackets (cf. [7]). For basic concepts, we refer to [1, $\S 4]$ and the literature cited there, and to $[8]$.

Let ${ }_{D} B_{E}$ be a bimodule over skew-fields $D$ and $E$. The left and right duals of $B$ are defined respectively by

$$
B^{*(-1)}:=\operatorname{Hom}_{D}(B, D), \quad B^{* 1}:=\operatorname{Hom}_{E}(B, E) .
$$

These are $(E, D)$-bimodules. Inductively, we define $B^{* 0}:=B$ and

$$
B^{*(-n-1)}:=\left(B^{*(-n)}\right)^{*(-1)}, \quad B^{*(n+1)}:=\left(B^{* n}\right)^{* 1}
$$

for $n \in \mathbb{N}$. A bimodule $B$ is said to be of finite dualization [7] if

$$
\left(B^{* m}\right)^{* n} \cong B^{*(m+n)}
$$

is an isomorphism of bimodules for all $m, n \in \mathbb{Z}$. An equivalent condition says that the dimension sequence

$$
a_{n}:= \begin{cases}\operatorname{dim}_{D} B^{* n} & \text { for } n \in \mathbb{Z} \text { even } \\ \operatorname{dim}_{E} B^{* n} & \text { for } n \in \mathbb{Z} \text { odd }\end{cases}
$$

is finite.

For a bimodule ${ }_{D} B_{E}$ of finite dualization, consider the hereditary artinian ring

$$
H(B):=\left(\begin{array}{cc}
E & 0 \\
B & D
\end{array}\right) .
$$

Any object of $H(B)$-mod, the category of finitely generated left $H(B)$ - 
modules, is given by a pair $\left(\begin{array}{l}X \\ Y\end{array}\right)$ consisting of a finite-dimensional left $E$ vector space $X$ and a finite-dimensional left $D$-vector space $Y$, together with a $D$-linear map $B \otimes_{E} X \rightarrow Y$. The dimension vector $\left(\begin{array}{l}x \\ y\end{array}\right)$ of $\left(\begin{array}{l}X \\ Y\end{array}\right)$, given by

$$
x:=\operatorname{dim}_{E} X, \quad y:=\operatorname{dim}_{D} Y,
$$

describes the corresponding element in the Grothendieck group $K_{0}(H(B))$. The dimension vectors of the indecomposable projective $H(B)$-modules $P_{0}:=\left(\begin{array}{l}0 \\ D\end{array}\right)$ and $P_{1}:=\left(\begin{array}{l}E \\ B\end{array}\right)$ are $\omega_{0}:=\left(\begin{array}{l}0 \\ 1\end{array}\right)$ and $\omega_{1}:=\left(\begin{array}{c}1 \\ a_{0}\end{array}\right)$, respectively. Using the Auslander-Reiten translate $\tau$, we define, more generally,

$$
P_{n}:= \begin{cases}\tau^{-n / 2} P_{0} & \text { for } n \in \mathbb{N} \text { even } \\ \tau^{-(n-1) / 2} P_{1} & \text { for } n \in \mathbb{N} \text { odd }\end{cases}
$$

Then

$$
\operatorname{Hom}_{H(B)}\left(P_{n}, P_{n+1}\right) \cong B^{* n} .
$$

Hence, by (31), the preprojective component of $H(B)$ looks as follows:

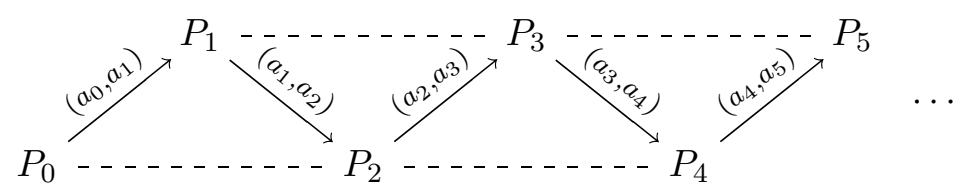

In particular, the dimension vector $\omega_{n}$ of $P_{n}$ satisfies

$$
\omega_{n-1}+\omega_{n+1}=a_{n} \omega_{n} .
$$

The indecomposable injective $H(B)$-modules are $I_{1}:=\left(\begin{array}{c}E \\ 0\end{array}\right)$ and $I_{2}:=\left(\begin{array}{c}B^{*(-1)} \\ D\end{array}\right)$, with structure maps $B \otimes_{E} E \rightarrow 0$ and $B \otimes_{E} B^{*(-1)} \rightarrow D$, respectively. Therefore, we have

$$
\operatorname{Hom}_{H(B)}\left(I_{2}, I_{1}\right) \cong B^{*(-2)} .
$$

So the preinjective component of $H(B)$ is of the form

(39) $\cdots$

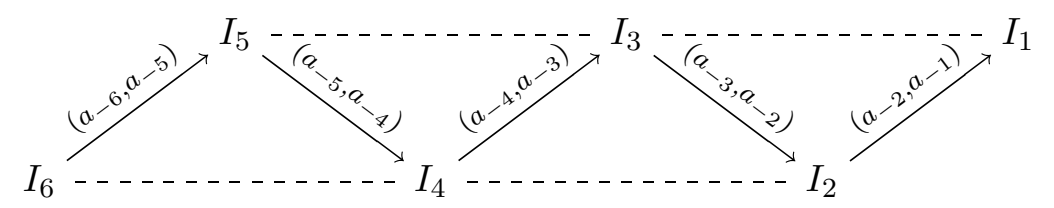

Furthermore, we have

$$
\operatorname{Ext}_{H(B)}^{1}\left(I_{1}, P_{0}\right) \cong B^{*(-1)}
$$

In fact, an extension of $P_{0}$ by $I_{1}$ amounts to a commutative diagram

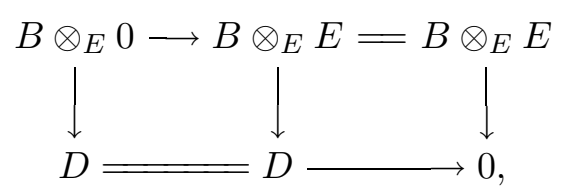

whence $\operatorname{Ext}_{H(B)}^{1}\left(I_{1}, P_{0}\right) \cong \operatorname{Hom}_{D}(B, D)=B^{*(-1)}$. 
In the derived category $D^{b}(H(B)$-mod), the two components (36) and (39) of the Auslander-Reiten quiver of $H(B)$ make up one component of one-termed complexes if the preinjective indecomposables are modified by a shift of one step to the right. By (40), the unified component looks as follows:

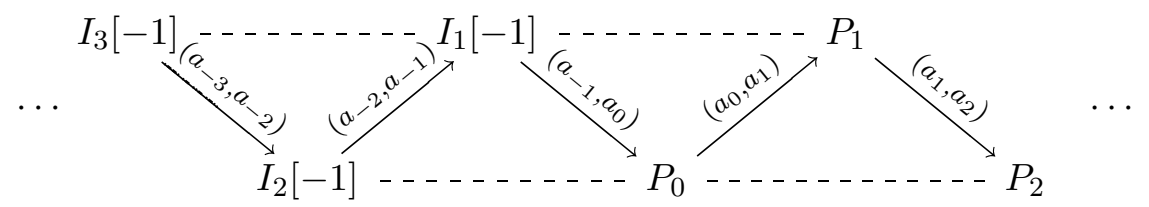

As an element of the Grothendieck group of $D^{b}(H(B)$-mod $)$, the dimension vector $\omega_{-n}$ of $I_{n}[-1]$ is negative for all $n>0$. In particular, we have $\omega_{-1}=$ $\left(\begin{array}{c}-1 \\ 0\end{array}\right)$ and $\omega_{-2}=\left(\begin{array}{c}-a_{-1} \\ -1\end{array}\right)$. Therefore, (37) holds for all $n \in \mathbb{Z}$. By Theorem 1 , the dimension sequence (31) defines a skew-symmetric unimodular bracket of signature 1, and (37) implies that $\left(\omega_{n}\right)_{n \in \mathbb{Z}}$ is the corresponding Farey sequence.

Thus we have shown that every bimodule ${ }_{D} B_{E}$ gives rise to a skewsymmetric unimodular bracket via (31), such that the dimension vectors of the combined preprojective-preinjective component of $D^{b}(H(B)$-mod) coincide with the vectors of the associated Farey sequence. Now we will show that all the values $[a, b]$ of the bracket are significant for the representation theory of $H(B)$.

To this end, we consider the $\left(H(B), H\left(B^{* 1}\right)\right)$-bimodule $T:=P_{2} \oplus P_{1}$. Explicitly, $P_{2}$ is given by the right-hand map of the short exact sequence

$$
D \hookrightarrow B \otimes_{E} B^{* 1} \rightarrow C .
$$

Here the embedding $D \hookrightarrow B \otimes_{E} B^{* 1}$ comes from the identification $B \otimes_{E} B^{* 1}$ $=\operatorname{End}_{E} \mathrm{op}(B)$. Thus

$$
T=\left(\begin{array}{cc}
B^{* 1} & E \\
C & B
\end{array}\right) .
$$

Since $T$ is a tilting module (see [12]), $T$ defines an adjunction

$$
H\left(B^{* 1}\right)-\bmod \underset{C^{+}}{\stackrel{C^{-}}{\rightleftarrows}} H(B)-\bmod
$$

with $C^{-}=T \otimes_{H\left(B^{* 1}\right)}-$ and $C^{+}=\operatorname{Hom}_{H(B)}(T,-)$. These functors coincide with the reflection functors $[2,7]$, defined as follows. Note first that for any $X \in E$-mod, there is a natural identification

$$
B \otimes_{E} X=\operatorname{Hom}_{E}\left(B^{* 1}, E\right) \otimes_{E} X=\operatorname{Hom}_{E}\left(B^{* 1}, X\right) .
$$

For $\left(\begin{array}{l}X \\ Y\end{array}\right) \in H(B)$-mod, given by $f: B \otimes_{E} X \rightarrow Y$, the $H\left(B^{* 1}\right)$-module $C^{+}\left(\begin{array}{c}X \\ Y\end{array}\right)$ is given by the adjoint map $B^{* 1} \otimes_{D} Z \rightarrow X$ of the kernel ker $f$ : 
$Z \longmapsto B \otimes_{E} X=\operatorname{Hom}_{E}\left(B^{* 1}, X\right)$. Similarly, for $\left(\begin{array}{l}U \\ V\end{array}\right) \in H\left(B^{* 1}\right)$-mod, given by $g: B^{* 1} \otimes_{D} U \rightarrow V$, the $H(B)$-module $C^{-}\left(\begin{array}{l}U \\ V\end{array}\right)$ is given by the cokernel $B \otimes_{E} V \rightarrow W$ of the adjoint map $\check{g}: U \rightarrow \operatorname{Hom}_{E}\left(B^{* 1}, V\right)=B \otimes_{E} V$.

The explicit form of $C^{+}$and $C^{-}$shows that $C^{-} C^{+} M \cong M$ holds for indecomposable $M \nsubseteq P_{0}$, while $C^{+} P_{0}=0$. Similarly, $C^{+} C^{-} N \cong N$ holds for indecomposable $N \in H\left(B^{* 1}\right)$ - mod with $N \approx J_{0}:=\left(\begin{array}{l}D \\ 0\end{array}\right)$. Now it is well-known that tilting modules gives rise to derived equivalences [4]. For the artinian rings $H(B)$, we thus have an equivalence

$$
H: D^{b}(H(B)-\mathbf{m o d}) \stackrel{\sim}{\longrightarrow} D^{b}\left(H\left(B^{* 1}\right) \text {-mod }\right) .
$$

The object map of $H$ is given by

$$
H(M)=\operatorname{Hom}_{D^{b}(H(B) \text {-mod })}(T, M) \cong \begin{cases}C^{+} M & \text { for } M \nsubseteq P_{0}, \\ J_{0}[-1] & \text { for } M \cong P_{0},\end{cases}
$$

for $M \in H(B)$-mod. Here, the case $M \cong P_{0}$ results from the fact that $\operatorname{Ext}_{H(B)}^{1}\left(P_{2}, P_{0}\right) \cong D$. So the component $(41)$ of $D^{b}(H(B)$-mod) is mapped onto the corresponding preprojective-preinjective component of the derived category $D^{b}\left(H\left(B^{* 1}\right)\right.$-mod $)$. With regard to the preprojective indecomposables in these components, the equivalence (46) amounts to a shift of one step to the left.

On the level of dimension vectors, $H$ induces the following bijection:

$$
H\left(\begin{array}{l}
x \\
y
\end{array}\right)=\left(\begin{array}{c}
a_{0} x-y \\
x
\end{array}\right) .
$$

In particular, this gives a bijective correspondence between the Farey sequences:

$$
H\left(\begin{array}{c}
{[n, 0]} \\
{[n,-1]}
\end{array}\right)=\left(\begin{array}{c}
{[n, 1]} \\
{[n, 0]}
\end{array}\right)
$$

Thus we have proved

Theorem 3. Let $D, E$ be skew-fields. For any $(D, E)$-bimodule $B$ of finite dualization, the dimension sequence (31) defines a unique skewsymmetric unimodular bracket of signature 1 with $a_{n}=[n, \infty]$ for $n \in \mathbb{Z}$. The preprojective or preinjective modules over the hereditary artinian ring (32) form an Auslander-Reiten component (41) of the derived category $D^{b}(H(B)$-mod), and the dimension vectors of the indecomposables of this component constitute the Farey sequence of the bracket. The horizontal translations of the bracket correspond to tiltings of $H(B)$.

REMARKS. 1. If we consider right $H(B)$-modules instead of left modules, the dimension sequence (31) turns into the sequence $\left(a_{1-n}\right)$, and the Farey 
sequence is changed into

$$
\omega_{n}^{\prime}=\left(\begin{array}{l}
{[2,1-n]} \\
{[1,1-n]}
\end{array}\right) .
$$

In other words, the Farey sequence of $H(B)^{\text {op }}$ is obtained from the $H(B)$ modules $P_{1}, P_{2}$ via successive tilting.

2. Theorem 3 implies that $H(B)$ is representation-finite if and only if the associated bracket is periodic. Since the terms $a_{n}$ of the dimension sequence (31) are all positive, it follows that the dimension sequences of representation-finite rings $H(B)$ can be described by triangulated polygons. For example, a hereditary artinian ring of type $\mathrm{G}_{2}$ corresponds to the triangulated hexagon (27). However, up to cyclic permutation, there are three other triangulated hexagons
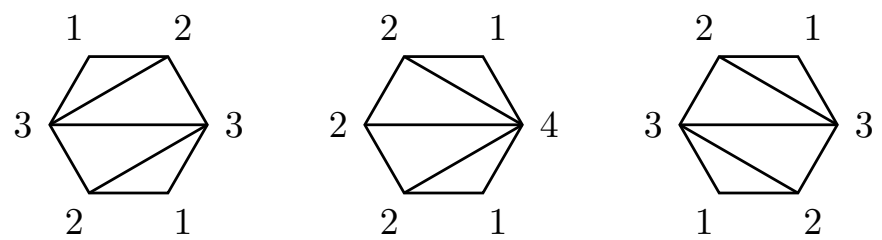

which lead to dimension sequences of type $\mathrm{I}_{2}(6)$. In contrast to $\mathrm{G}_{2}$, they are non-cristallographic in the sense that the Auslander-Reiten translate $\tau$ induces a non-linear map on the Grothendieck group of $D^{b}(H(B)$-mod). In this respect, the Coxeter diagram $\mathrm{I}_{2}(6)$ should be distinguished from $\mathrm{G}_{2}$.

\section{References}

[1] D. J. Benson, Representations and Cohomology, I, Cambridge Stud. Adv. Math. 30, Cambridge Univ. Press, 1991.

[2] I. N. Bernstein, I. M. Gelfand and V. A. Ponomarev, Coxeter functors and Gabriel's theorem, Uspekhi Mat. Nauk 28 (1973), 19-33 (in Russian); English transl.: Russian Math. Surveys 28 (1973), 17-32.

[3] K. Bongartz and P. Gabriel, Covering spaces in representation theory, Invent. Math. 65 (1982), 331-378.

[4] E. Cline, B. Parshall and L. Scott, Derived categories and Morita theory, J. Algebra 104 (1986), 397-409.

[5] J. H. Conway and H. S. M. Coxeter, Triangulated polygons and frieze patterns, Math. Gaz. 57 (1973), 87-94.

[6] H. S. M. Coxeter, Frieze patterns, Acta Arith. 18 (1971), 297-310.

[7] P. Dowbor, C. M. Ringel and D. Simson, Hereditary Artinian rings of finite representation type, in: Representation Theory, II (Ottawa, 1979), Lecture Notes in Math. 832, Springer, 1980, 232-241.

[8] D. Happel, Triangulated Categories in the Representation Theory of Finite Dimensional Algebras, London Math. Soc. Lecture Note Ser. 119, Cambridge Univ. Press, 1988.

[9] D. Happel and C. M. Ringel, Construction of tilted algebras, in: Representations of Algebras (Puebla, 1980), Lecture Notes in Math. 903, Springer, 1981, 125-144. 
[10] G. H. Hardy and E. M. Wright, An Introduction to the Theory of Numbers, 4th ed., Oxford Univ. Press, London, 1975.

[11] D. A. Klarner, Correspondences between plane trees and binary sequences, J. Combin. Theory 9 (1970), 401-411.

[12] Y. Miyashita, Tilting modules of finite projective dimension, Math. Z. 193 (1986), $113-146$.

[13] Th. Motzkin, Relations between hypersurface cross ratios, and a combinatorial formula for partitions of a polygon, for permanent preponderance, and for non-associative products, Bull. Amer. Math. Soc. 54 (1948), 352-360.

[14] J.-Y. Shi, The Kazhdan-Lusztig Cells in Certain Affine Weyl Groups, Lecture Notes in Math. 1179, Springer, 1986.

[15] - Sign types corresponding to an affine Weyl group, J. London Math. Soc. 35 (1987), 56-74.

[16] - The number of $\oplus$-sign types, Quart. J. Math. 48 (1997), 93-105.

[17] R. P. Stanley, Enumerative Combinatorics, I, Cambridge Stud. Adv. Math. 49, Cambridge Univ. Press, 1997.

Institut für Algebra und Zahlentheorie

Universität Stuttgart

Pfaffenwaldring 57

D-70550 Stuttgart, Germany

E-mail: rump@mathematik.uni-stuttgart.de 